\title{
Multiple species atom source for laser-cooling experiments
}

\author{
C. A. Stan and W. Ketterle \\ Department of Physics, MIT-Harvard Center for Ultracold Atoms, and Research Laboratory of Electronics, \\ MIT, Cambridge, Massachusetts 02139
}

(Dated: June 2, 2005)

\begin{abstract}
We describe the design of a single beam, multiple species atom source in which the flux of any component can be separately adjusted. Using this design we have developed a ${ }^{23} \mathrm{Na}-{ }^{6} \mathrm{Li}$ atom source for ultracold atom experiments. The fluxes of lithium and sodium are independently tunable, allowing operation as a single ${ }^{23} \mathrm{Na}$ or ${ }^{6} \mathrm{Li}$ source as well as a double source with equal atomic fluxes in each component.

PACS numbers: 03.75.F, 07.77.Gx, 32.80.Pj, 39.10.+j
\end{abstract}

\section{INTRODUCTION}

In recent years a number of frontier atomic physics experiments have used more than one atomic species. The most precise measurement of the electrical dipole moment of the electron to date [1] used two atoms to suppress systematic errors. Ultracold mixtures of two atomic species are used for sympathethic cooling of fermions [2, 3, 4], for the study of atomic Bose-Fermi mixtures [5], and of interspecies Feshbach resonances 6, 7]. Simultaneous laser cooling of the atomic components is used to study interspecies collisions 8, 9], and to produce ultracold heteronuclear molecules [10] which might be used for quantum computation 11] or the observation of exotic many-body states [12, 13].

Vapor cells and atomic beams are the most common atom sources for cold atom experiments. In a vapor cell, an atomic trap is loaded from background gas. Higher background gas pressures results in faster loading rates, but above an optimum pressure losses due to collisions with the background gas increase as fast as the loading rate. Multispecies vapor cells have been used to generate a slow beam of rubidium and cesium atoms [8].

Loading an atomic trap from a collimated beam of atoms is compatible with ultrahigh vacuum and avoids background gas collisions. The flux of atoms slow enough to be captured by the trap can be considerably increased compared to a thermal beam by using a Zeeman slower [14]. The Zeeman slower system has higher loading rates than a vapor cell.

A multiple-species experiment can be designed with multiple independent beam sources, but this increases its complexity. We have developed a two-species atomic oven for ${ }^{23} \mathrm{Na}$ and ${ }^{6} \mathrm{Li}$ which works with a single Zeeman slower to load ${ }^{23} \mathrm{Na}$ and ${ }^{6} \mathrm{Li}$ magneto-optical traps (MOTs) simultaneously.

The paper is organized as follows: in Sec. II we present the general design and operation criteria for a multiple species oven, and in Sec. III the construction of the sodium-lithium source. We describe the operation of this source and its performance in Sec. IV, and we discuss in Sec. $\mathrm{V}$ the applicability of the sodium-lithium design to other atomic species used in laser-cooling experiments.

\section{GENERAL DESIGN CRITERIA FOR A MULTIPLE SPECIES SOURCE}

Effusive ovens [15] are simple and efficient atom sources. They consist of an evacuated reservoir chamber in which the desired species is stored in solid or liquid form, and is in equilibrium with its vapor. The vapor effuses through a small opening towards the experiment. The flux is easily controlled by changing the oven temperature and thus the equilibrium vapor pressure in the reservoir.

The relation between the flux and the vapor pressure is linear as long as the vapor pressure is low enough that the flow through the oven nozzle is molecular. Equivalently, the mean free path at the vapor pressure is bigger than the nozzle size. Operation beyond the linear regime results in viscous or supersonic flows, characterized by higher total atom fluxes and by a depletion of the low velocity tail of the Maxwell distribution. Zeeman slowing usually captures the low-velocity tail and therefore requires operation of the oven in the linear regime.

If multiple atomic or molecular species must be present in the beam, loading a single reservoir with more than one pure substance is in general not appropriate. The vapor pressures at the same temperature are likely to be different. The ratio of fluxes is then fixed at approximately the ratio of the vapor pressures, which has a weak dependence on temperature. In the case of our experiment, sodium has a vapor pressure three orders of magnitude higher than lithium at the same temperature, meaning that at optimum sodium flux the lithium flux is too low, and at the optimum lithium flux the sodium consumption rate is impractical.

A solution to this problem is the use of multiple reservoirs, each holding a pure component, connected to a mixing chamber (Fig. 11). The design requirements for all reservoirs is that they should deliver their component to the mixing chamber at an adjustable rate. In steady state, the mixing chamber should not absorb the components. Then, the flux of one species through the oven nozzle equals the flux through the mixing nozzle, and can be tuned by changing the temperature of the reservoir.

For simplicity we will assume that the pressures in the reservoirs are much bigger than the pressure in the mix- 


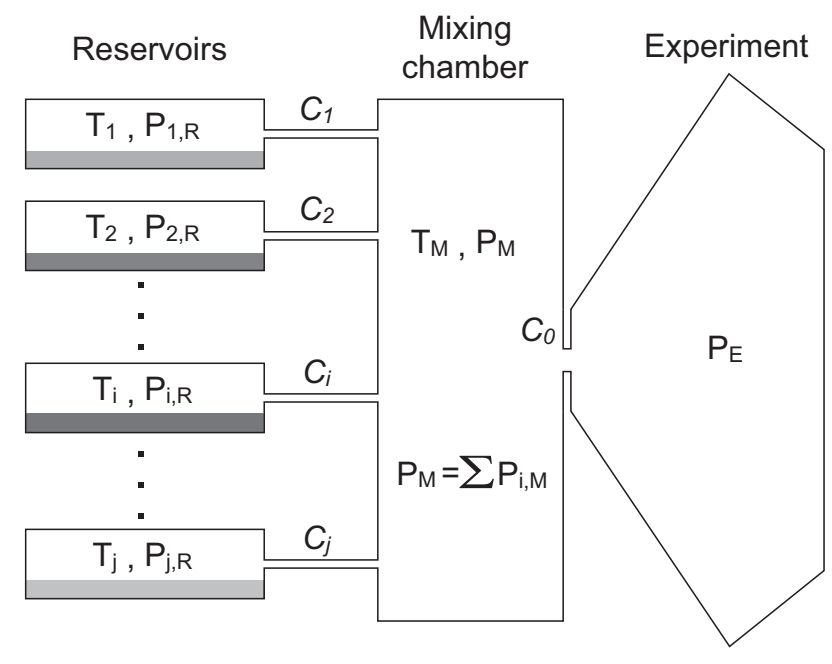

FIG. 1: Design of a multiple species oven. Reservoirs containing pure components are connected to a mixing chamber through mixing nozzles. The mixing chamber is connected to the experiment through the oven nozzle. $P_{i, R}$ are the saturated vapor pressures at the reservoir temperatures $T_{i}$. In the mixing chamber a gaseous mixture at temperature $T_{M}$ is formed, with partial pressures $P_{i, M} . P_{E}$ is the pressure in the experiment chamber. Nozzle vacuum conductances are shown in italics. For proper operation, $P_{i, R} \gg P_{i, M} \gg P_{E}$.

ing chamber, which in its turn is much bigger than the pressure outside the oven. Such pressure ratios are also the best choice for oven operation, as it will be seen below.

The throughput of the $i$ th component, $Q_{i, R}$, from the $i$ th reservoir to the mixing chamber is given by

$$
Q_{i, R}=C_{i}\left(P_{i, R}-P_{i, M}\right) \simeq C_{i} P_{i, R}
$$

where $C i$ is the conductance of the $i$ th mixing nozzle, $P_{i, R}$ the vapor pressure in the $i$ th reservoir, and $P_{i, M}$ the partial pressure in the mixing chamber. Assuming that the reservoirs are not contaminated with different species, and that $P_{i, M}$ is smaller than the saturated vapor pressure in the mixing chamber, the throughput of the component from the oven to the experiment is given by

$$
Q_{i, T}=C_{0} P_{i, M}=Q_{i, R}-P_{i, M} \sum_{j \neq i} C_{j}
$$

where $C_{0}$ is the vacuum conductance of the oven nozzle and $P_{i, M}$ is the partial pressure of component $i$ in the mixing chamber. The second term in the sum denotes the loss of component $i$ by backflow into other reservoirs. It can be made negligible if all the mixing nozzle conductances $C_{j}$ are much smaller than $C_{0}$.

In steady state operation, assuming that all mixing nozzles have conductances much smaller than the oven nozzle,

$$
Q_{i, T}=Q_{i, R}=C_{i} P_{i, R}
$$

and the flux of component $i$ from the oven can be tuned by heating the reservoir in the same way as for a simple effusive oven.

Backflow of other species from the mixing chamber into the reservoir is not desirable, for two reasons. First, these other species will be lost into the reservoir rather than making the effusive beam. Second, different species could react with or dissolve into the pure substance loaded in the reservoir, reducing its vapor pressure 16.

Reducing the backflow to negligible levels and even complete suppression can be achieved by a proper design of the mixing nozzles. Backflow occurs through diffusion against the constant stream of atoms or molecules coming from the reservoir. If this stream is sufficiently fast it will blow away diffusing components before they could reach the reservoir.

We have estimated the parameters of the mixing nozzle by requiring that in the nozzle the diffusion speed of component $j$ into component $i$ is smaller than the macroscopic speed of the stream. For a nozzle made of a long cylindrical tube, the approximate condition for suppression is

$$
\lambda_{j, P_{i, R}}<d
$$

where $\lambda_{j, P_{i, R}}$ is the mean free path of species $j$ in the gas of $i$ at reservoir $i$ pressure $P_{i, R}$, and $d$ is the nozzle diameter. An equivalent statement is that the flow in the mixing nozzle should be viscous or at least in the intermediate regime.

Chemical reactions and solution formation is also a concern for the mixing chamber, but negative effects can be diminished by raising the temperature of the chamber. As long as the partial pressures of different components are below their saturated vapor pressure, condensation will not occur, and the rate of chemical reactions will be significantly decreased. The guidelines for temperature settings are completed by the condition that the nozzles should have the highest temperatures in the oven to avoid clogging.

\section{THE SODIUM-LITHIUM ATOM SOURCE}

A schematic of our ${ }^{23} \mathrm{Na}-{ }^{6} \mathrm{Li}$ oven is given in Fig. 2 The oven follows the basic design principles described above, but its construction was simplified by putting the lithium in the mixing chamber rather than in its own reservoir. This modification was necessary to keep the maximum oven temperature at the specified operation limit of ConFlat knife-edge vacuum flanges, $450{ }^{\circ} \mathrm{C}$. The vapor pressure required for proper operation of a lithium reservoir must be tens of times bigger than the vapor pressure in the mixing chamber, and can be achieved only at higher temperatures. Building the oven from ConFlat parts makes it compatible with the rest of our vacuum chamber, and allows easy assembly and disassembly during alkali reloading. 


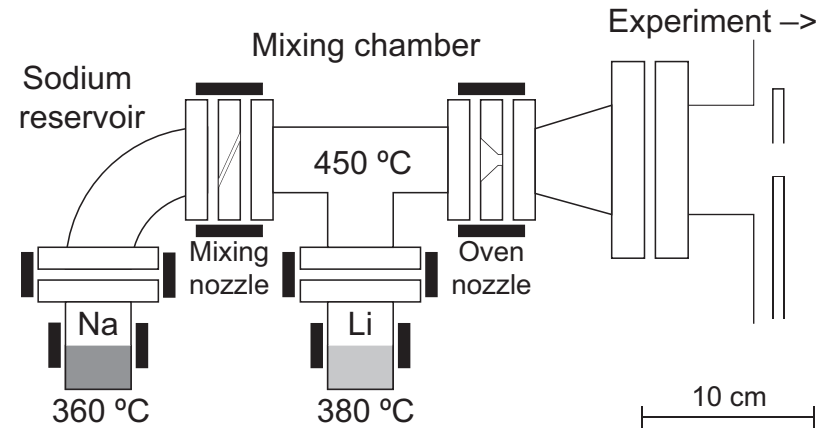

FIG. 2: Exploded cross section view, to scale, of the ${ }^{23} \mathrm{Na}$ $-{ }^{6} \mathrm{Li}$ source. The oven is built from 2.75 inch ConFlat fittings, made from 316 SS. Alkali receptacles are capped half nipples, and the nozzles are machined from double-sided blanks. The $2 \mathrm{~mm}$ diameter mixing nozzle is angled to achieve a length of $32 \mathrm{~mm}$, and the thin oven nozzle has a diameter of $4 \mathrm{~mm}$. A copper plate with a $7.5 \mathrm{~mm}$ hole, shown at the right edge of the figure, acts as a collimation aperture. Black rectangles represent the six resistive band heaters used for temperature adjustment. The shown temperatures of the alkali receptacles and of the mixing chamber are typical for double species operation.

Since the lithium is placed in the mixing chamber, chemical reaction and alloy formation are possible. The binary phase diagram of sodium and lithium [17] exhibits immiscibility regions. Above lithium's melting temperature, $180.6^{\circ} \mathrm{C}$, the mixture is liquid at all concentrations, but it can phase separate into sodium-rich and lithiumrich liquids. Above the critical temperature, $303.2{ }^{\circ} \mathrm{C}$, the sodium and the lithium are fully miscible.

A small amount of sodium always dissolves into the lithium during two species operation. Above the critical temperature there is only one liquid phase, a solution of sodium in lithium. The concentration of sodium can be estimated by assuming that the solution is ideal and thus obeys Raoult's law. Raoult's law states that the vapor pressure of the solute is smaller than the saturated vapor pressure by the atomic concentration of the solute in the solvent. The vapor pressure of sodium is three orders of magnitude higher than lithium's at the same temperature. In an alloy above which both elements have equal partial vapor pressures, the atomic concentration of sodium is then only $0.1 \%$. The sodium-lithium mixture exhibits tendency towards phase separation. Therefore a positive deviation from Raoult's law is expected [18], and the real sodium concentration is smaller than our estimate. Since the sodium concentration is small, lithium vapor pressure is not decreased significantly by the presence of sodium.

The sodium-lithium oven is built from 2.75 inch ConFlat fittings. The presence of lithium, which reacts with metals more aggressively than other alkali, and the relatively high operating temperatures required the use of non-standard materials for the fittings and the gaskets.
Copper gaskets are the standard choice, but when heated to $400-450{ }^{\circ} \mathrm{C}$ they can bond to the knife edges, making disassembly difficult. Annealed nickel gaskets were chosen for their high temperature performance and for their good compatibility with molten alkali metals.

Nickel gaskets, even when fully annealed, are harder than copper gaskets, and their properties vary among manufacturers and different batches. As a result, reliable sealing and resealing was difficult when we used standard ConFlat fittings made from type 304 stainless steel. The knife edge dulls after only a few reseals, probably because of annealing of type 304 when it is cycled from room temperature to $400-450{ }^{\circ} \mathrm{C}$.

We have overcome this problem by using slightly modified fittings made from type 316 stainless steel. A chromium-nickel steel, type 316's composition differs from type 304's by the addition of $2-3 \%$ percent molybdenum. It is offered as a material option by vacuum manufacturers for its better high-temperature strength. We have found that type 316 fittings were indeed superior to standard type 304 fittings between 400 and 450 ${ }^{\circ} \mathrm{C}$ and could be resealed multiple times.

The fittings were modified by removing a $0.127 \mathrm{~mm}$ layer of material from the flange faces. By doing this the knife edge recession is reduced and the knife can cut deeper into the nickel gaskets, which we often found to be thinner than industry specifications. The modified fittings were manufactured by A\&N Corporation [19].

The oven nozzle has a diameter of $4 \mathrm{~mm}$, equal to the narrowest collimating aperture, a differential pumping tube. The mixing nozzle is a $2 \mathrm{~mm}$ diameter, 32 $\mathrm{mm}$ long tube angled with respect to the axis of the machine. Both nozzles were machined in double-sided ConFlat blanks.

For heating, band heaters are placed around flanges and around alkali receptacles. We have used custom MiPlus heaters manufactured by TEMPCO [20]. They can be operated at temperatures up to $760{ }^{\circ} \mathrm{C}$, and have incorporated K-type thermocouples, ensuring reproducible temperature readout. The heater powers are $100 \mathrm{~W}$ for the alkali receptacles, $150 \mathrm{~W}$ for the receptacle flanges, and $300 \mathrm{~W}$ for the nozzle flanges.

The heaters are controlled by commercial temperature controllers. The oven is thermally insulated by wrapping first ceramic fiber tape to form a $1 \mathrm{~cm}$ thick layer of insulation. Two or three layers of household use aluminum foil are wrapped on top of this layer. Temperature stability is approximately $0.1{ }^{\circ} \mathrm{C}$.

The oven is connected to a section of our apparatus in which the pressure is $1 \times 10^{-8}$ Torr. In this section the beam is collimated to $5 \times 10^{-5}$ sr divergence by a differential pumping tube with an inner diameter of 4 $\mathrm{mm}$. 


\section{OPERATION AND PERFORMANCE}

The oven is loaded with $25 \mathrm{~g}$ of ${ }^{23} \mathrm{Na}$ and $10 \mathrm{~g}$ of isotopically enriched ${ }^{6} \mathrm{Li}$ (95\% purity). Sodium is commercially available in sealed glass ampoules which we break just before loading. Enriched ${ }^{6} \mathrm{Li}$ is available as chunks under mineral oil, and has to be cleaned prior to loading. We had the ${ }^{6} \mathrm{Li}$ cleaned and repackaged into sealed glass ampoules by a materials preparation laboratory [21].

The target alkali temperatures are achieved by setting the receptacle heaters to these temperatures, $360{ }^{\circ} \mathrm{C}$ for sodium and $380{ }^{\circ} \mathrm{C}$ for lithium. The heaters around the flanges of the receptacles are set to $20{ }^{\circ} \mathrm{C}$ above the alkali temperature for a smooth thermal gradient from the alkali receptacles to the nozzles. Nozzle heaters are both set to $450{ }^{\circ} \mathrm{C}$. The nozzles are the hottest areas in the oven to prevent their clogging. When the experiment is not running, nozzle heaters are kept on while the other heaters are turned off. From this idle state, it takes less then 10 minutes to heat the oven to its operating temperatures.

The mixing nozzle operates in the viscous flow regime. Its conductance, $0.08 \mathrm{~L} / \mathrm{s}$, is difficult to calculate in this regime, and was measured from the sodium consumption rate. At $450{ }^{\circ} \mathrm{C}$ the calculated molecular flow conductance of the oven nozzle is $2.6 \mathrm{~L} / \mathrm{s}$ for sodium and $5 \mathrm{~L} / \mathrm{s}$ for lithium. This results in a ratio of sodium pressures in the reservoir and the mixing chamber of approximately 30. The partial pressures in the mixing chamber are $4 \times 10^{-3}$ Torr for sodium and $4 \times 10^{-5}$ Torr for lithium.

Alkali-alkali collision cross sections at room temperature are quite large, making the mean free paths in alkali vapor considerably shorter than those encountered in normal vacuum practice. We have estimated the cross sections using the Massey-Mohr formula 22]:

$$
\sigma=5 \times 10^{11}\left(C_{6} / v\right)^{2 / 5}
$$

where $\sigma$ is the collision cross-section in $\mathrm{cm}^{2}, C_{6}$ the Van der Waals coefficient in $\mathrm{erg} \cdot \mathrm{cm}^{6}$, and $v$ the speed in $\mathrm{cm} / \mathrm{s}$. For sodium we have used the experimental value of $C_{6}$ given in 23] and for lithium the theoretical calculation given in [24].

The estimated mean free path of lithium in the sodium reservoir is $0.1 \mathrm{~mm}$, satisfying the condition for backflow suppression given in the previous section. The estimated mean free paths for sodium and lithium in the mixing chamber are 1.7 and $2.8 \mathrm{~mm}$, slightly lower than required for molecular flow through the $4 \mathrm{~mm}$ oven nozzle. In this regime the oven nozzle conductance, calculated using conductance tables given in [16], is not significantly changed from its molecular flow value.

Total sodium atom flux, measured from the sodium consumption rate, is $1.6 \times 10^{17} \mathrm{~s}^{-1}$. A lithium flux of $3 \times 10^{15} \mathrm{~s}^{-1}$ was estimated from the vapor pressure. The fluxes in the collimated beam are approximately $10^{4}$ times lower. The limits on flux tuning are given by different factors for sodium and lithium. For sodium, the minimum operating temperature is approximately $200{ }^{\circ} \mathrm{C}$, the equilibrium temperature with only the nozzle heaters on. The upper limit, $360{ }^{\circ} \mathrm{C}$, is given by the requirement that the flow through the oven nozzle is molecular. For lithium the upper limit, $435{ }^{\circ} \mathrm{C}$, is given by the requirement that the nozzles are the hottest part of the oven. We have decided not to operate the lithium reservoir below the critical temperature, $303.2^{\circ} \mathrm{C}$, to avoid a possibly complicated dependence between reservoir temperature and atom flux. The atomic fluxes can be varied over three orders of magnitude, between $1.3 \times 10^{14} \mathrm{~s}^{-1}$ and $1.6 \times 10^{17} \mathrm{~s}^{-1}$ for sodium, and from $5 \times 10^{13} \mathrm{~s}^{-1}$ to $3 \times 10^{16} \mathrm{~s}^{-1}$ for lithium.

The Zeeman slower originally designed for sodium was modified for double operation by overlapping lithium laser slowing light with the sodium slowing light. With this double atom source we can load sodium, lithium, or overlapping double species MOTs. The lithium MOT traps $3 \times 10^{8}$ atoms and loads in approximately $4 \mathrm{~s}$. The sodium MOT traps $10^{10}$ atoms and loads in approximately $2 \mathrm{~s}$. The atom numbers given are accurate within a factor of 2 . For the double species MOT, the number of lithium atoms is reduced to approximately half, as measured from the drop in MOT fluorescence intensity. The number of sodium atoms is not changed.

The double species MOT provides an excellent starting point for further cooling the mixture into quantum degeneracy. Bose-Einstein condensates of ${ }^{23} \mathrm{Na}$ with $2 \times 10^{7}$ atoms and degenerate ${ }^{6} \mathrm{Li}$ Fermi gases with $5 \times 10^{7}$ atoms are routinely produced [25].

With a full $25 \mathrm{~g}$ sodium load, the oven operates continuously for 1200-1300 hours. We have estimated that the $10 \mathrm{~g}$ lithium load should last for 10000 hours at the maximum flux. Sodium changes are performed twice a year. We do not find any lithium deposits in the sodium reservoir, which proves that the mixing nozzle design suppresses lithium backflow. The low conductance mixing nozzle allows clean venting with high purity argon during the changes, and baking is not needed. The experiment can be run again within hours of sodium reloading.

Most of the effused sodium deposits on the collector plate and on the walls of the 4.5 inch ConFlat 6-way cross to which the oven is attached. Sodium needs to be cleaned every 2-3 years. The deposition occurs in readily accessible areas and the cleaning procedure takes at most a few days.

\section{DISCUSSION}

Efficient loading of a multiple-species MOT using a multiple species oven requires the use of a Zeeman slowing scheme which operates for all species. While laser beams with different frequencies can be easily overlapped, the solenoid coil which generates the spatially varying magnetic field is the same for all species.

It is possible to design a solenoid which will operate for more than one alkali atom. The most important parameter for the design of a Zeeman slower is the maximum 
acceleration of an atom interacting with a resonant laser beam:

$$
a_{\max }=\frac{\pi \hbar \Gamma}{m \lambda}
$$

where $\Gamma$ and $\lambda$ are the linewidth and the wavelength of the resonant light, and $m$ is the mass of the atom. In the case of alkali atoms, it is the atom mass which varies most from species to species. The maximum acceleration decreases from lithium to cesium.

The construction of the slowing coil defines the maximum speed of the atoms which can be slowed (the capture velocity), and the acceleration of the slowed atoms. This acceleration must be smaller than $a_{\max }$. Since $a_{\max }$ decreases with mass, a coil designed for a given alkali will work also for lighter species. However, at the same capture velocity and same temperature a smaller part of the Maxwell-Boltzmann distribution can be slowed for the lighter atom. In our experiment, the performance of sodium-designed coils at slowing lithium was satisfactory.

The use of single beam, multiple atom source can significantly simplify the design of a multiple species lasercooling experiment. The general design criteria given in Sec. II is applicable for any mixture of pure substances as long as chemical reactions do not occur between the components. The trichamber thallium-sodium oven described in [1], developed independently from ours, uses the same principle as our sodium-lithium source.

The ConFlat construction we adopted for our sodiumlithium oven does not require complicated machining and can be applied to many of the atomic isotopes used in laser-cooling experiments. The main limitation of the ConFlat design using 316SS parts and nickel gaskets is its maximum operating temperature. Multiple reassembly can be easily achieved at temperatures up to $400{ }^{\circ} \mathrm{C}$. We have been able to reassemble a flange heated to $500{ }^{\circ} \mathrm{C}$ and we have learned that the seals are reliable to at least $650{ }^{\circ} \mathrm{C}$, although they might not be resealable.

Given the typical atomic fluxes needed in laser-cooling experiments, partial pressures in the mixing chamber should be around $1 \times 10^{-3}$ Torr. Alkali isotopes of $\mathrm{K}$, $\mathrm{Rb}$ and $\mathrm{Cs}$ all have vapor pressures higher than sodium. $\mathrm{Ca}, \mathrm{Sr}$, and $\mathrm{Yb}$ isotopes which have been laser-cooled to high phase space densities [26, 27, 28] have vapor pressures comparable with lithium. The design described here should work for all these species.

\section{Acknowledgments}

This work was supported by the NSF, ONR, ARO, and NASA. We thank Michele Saba and Zoran Hadzibabic for a critical reading of the manuscript.
[1] B. C. Regan, E. D. Commins, C. J. Schmidt, and D. DeMille, Phys. Rev. Lett. 88, 071805 (2002).

[2] Z. Hadzibabic, C. A. Stan, K. Dieckmann, S. Gupta, M. W. Zwierlein, A. Görlitz, and W. Ketterle, Phys. Rev. Lett. 88, 160401 (2002).

[3] A. G. Truscott, K. E. Strecker, W. I. McAlexander, G. B. Partridge, and R. G. Hulet, Science 291, 2570 (2001).

[4] F. Schreck, L. Khaykovich, K. L. Corwin, G. Ferrari, T. Bourdel, J. Cubizolles, and C. Salomon, Phys. Rev. Lett. 87, 080403 (2001).

[5] G. Modugno, G. Roati, F. Riboli, F. Ferlaino, R. J. Brecha, and M. Inguscio, Science 297, 2240 (2002).

[6] C. A. Stan, M. W. Zwierlein, C. H. Schunck, S. M. F. Raupach, and W. Ketterle, Phys. Rev. Lett. 93, 143001 (2004).

[7] S. Inouye, J. Goldwin, M. L. Olsen, C. Ticknor, J. L. Bohn, and D. S. Jin, Phys. Rev. Lett. 93, 183201 (2004).

[8] N. Lundblad, D. C. Aveline, R. J. Thompson, J. M. Kohel, J. Ramirez-Serrano, W. K. Klipstein, D. G. Enzer, N. Yu, and N. Maleki, J. Opt. Soc. Am. B 21, 3 (2004).

[9] M. S. Santos, P. Nussenzveig, L. G. Marcassa, K. Helmerson, J. Flemming, S. C. Zilio, and V. S. Bagnato, Phys. Rev. A 52, R4340 (1996).

[10] J. P. Shaffer, W. Chalupczak, and N. P. Bigelow, Phys. Rev. Lett. 82, 11241127 (1999).

[11] D. DeMille, Phys. Rev. Lett. 88, 067901 (2002).

[12] K. Góral, L. Santos, and M. Lewenstein, Phys. Rev. Lett. 88, 170406 (2002).

[13] M. A. Baranov, M. S. Mar'enko, V. S. Rychkov, and G. V. Shlyapnikov, Phys. Rev. A 66, 013606 (2002).
[14] W. D. Phillips and H. Metcalf, Phys. Rev. Lett. 48, 596 (1982).

[15] K. J. Ross and B. Sonntag, Rev. Sci. Instrum. 66, 4409 (1995).

[16] S. Dushman, Scientific foundations of vacuum technique, pp. 710-722 (Wiley, New York, 1962).

[17] T. B. Massalski, H. Okamoto, P. R. Subramanian, and L. Kacprzak, Binary alloy phase diagrams, pp. 2448-2450 (ASM International, Materials Park, OH, 1990).

[18] H.-G. Lee, Chemical thermodynamics for metals and materials, pp. 83-86 (Imperial College Press, London, 1999).

[19] A\&N Corporation, Williston, FL, USA.

[20] TEMPCO Electric Heater Corporation, Wood Dale, IL, USA.

[21] Materials Preparation Center, Ames Laboratory, Ames, IA, USA.

[22] H. S. W. Massey and C. B. O. Mohr, Proc. R. Soc. London, Ser. A 144, 188 (1934).

[23] U. Buck and H. Pauly, Z. Phys. 185, 155 (1965).

[24] P. R. Fontana, Phys. Rev. 123, 1865 (1961).

[25] Z. Hadzibabic, S. Gupta, C. A. Stan, C. H. Schunck, M. W. Zwierlein, K. Dieckmann, and W. Ketterle, Phys. Rev. Lett. 91, 160401 (2003).

[26] T. Binnewies, G. G. Wilpers, U. Sterr, F. Riehle, and J. Helmcke, Phys. Rev. Lett. 87, 123002 (2001).

[27] H. Katori, T. Ido, Y. Isoya, and M. Kuwata-Gonokami, Phys. Rev. Lett. 82, 1116 (1999).

[28] Y. Takasu, K. Maki, K. Komori, T. Takano, K. Honda, M. Kumakura, T. Yabuzaki, and Y. Takahashi, Phys. Rev. Lett. 91, 040404 (2003). 